\title{
Omicron: Possible Origins and Accelerated Mutative Cycle Production
}

\author{
Serge Dolgikh ${ }^{10000-0001-5929-8954] ~}$ \\ Dept. of Information Technology, \\ National Aviation University, Kyiv
}

\begin{abstract}
A hypothesis on possible origins and development of the Covid-19 Omicron and similar variants with large number of mutations is proposed and discussed. Possible connections with natural immunity variability in the population is explored.
\end{abstract}

Keywords: Epidemiology, immunity, Covid-19, Omicron

\section{Omicron Variant}

A new variant of Covid-19 viral infection was detected in November 2021 in South African health jurisdictions [1,2]. At the time of writing, it is propagating globally, with cases reported in a number of national health jurisdictions in Europe, Asia and North America.

The variant has been noted to possess several distinct properties that will be of interest in this analysis. The first is an unusually high number of mutations [3], raising a number of concerns as discussed in the research community and the media, but also appearing to be less likely to emerge randomly in a single "burst" of mutations. The second one, at this time pointed tentatively by anecdotal evidence, is a possible ability to avoid the immune response in fully vaccinated patients, supported by significant prevalence of fully vaccinated in the identified cases of the variant $[4,5]$.

\section{Induced Immunity and Counter-Immune Selection}

Interactions of immunity characteristics (immunity profiles) induced by mass vaccinations based on particular elements of the viral agent, such as specific proteins, and a natural variation in the immunity in the population was discussed in [6].

In particular, it was noted that in certain infectious environments, including those with a combination of a highly mutable viral agent and a "standard" immunity profile programmed to respond to specific antigens, induced in the population by indiscriminate mass immunization, a natural mutability of the agent can produce random variations with positive selection to counter the induced immunity response. It can be illustrated as a higher mutative pressure on the regions of the viral agents that are detected by the immune response induced by vaccines associated with preferred selection of less detectable mutations. The resulting mutability vector can be seen as to counter 
the immune response and produce a higher number of mutations in the directions less prone to detection and neutralization, resulting in a higher resistance to induced immunity.

These conclusions combined with the initial observations of the properties of the new variant suggest a hypothesis on its possible origin that can be evaluated as more evidence emerges on the properties and behavior of the variant.

\section{$3 \quad$ Omicron Origins: a Positive Mutative Selection Loop?}

As was noted earlier, an unusually high number of mutations may suggest that such a combination of viral change would be less likely to emerge in a single random event, based on statistical considerations. Another possibility is a process that can lead to accumulation of several or multiple changes, positively selected to improve infectivity in both vaccinated and non-vaccinated populations.

One such possibility is a continuous positive selection cycle with two interacting populations pools, one serving as a reservoir sustaining the mutating agent, and the other, as an "engine" producing new mutations (Figure 1).
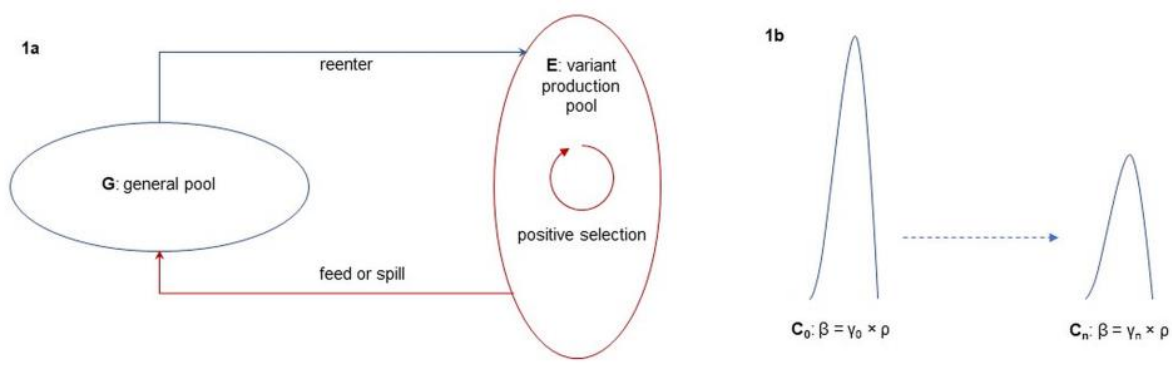

Fig.1 Positive mutation production loop

The cycle begins with a mutation with somewhat higher resistance in a vaccinated environment, for example, a change in the specific region targeted by the vaccine that can occur randomly. It is carried and sustained in the general pool (G, Fig. 1a) and eventually reenters back into the vaccinated environment $(\mathrm{E})$, a confirmed possibility based on multiple reports of outbreaks in highly vaccinated areas, such as flights, hospitals, sports teams and others [7,8].

The cycle then repeats, producing a positive reinforcement loop: the changes with improved resistance are selected and sustained in the general pool, while those that produce less resistant mutations, suppressed. The resulting variants would have acquired multiple positive mutations (for the viral agent) that would otherwise be strongly suppressed by statistical factors. The process slows down and eventually terminates as one of, or both pools reach the state of group immunity.

The scenario is controlled by several parameters, such as the size of the pools that has to be sufficiently large to allow a number of cycles before the loop process terminates, the rate of interaction between the pools (contact rate, $\rho$ ) and the cross-pool transmission factor, $\gamma$ that is, the infectivity rate on a contact between members of the 
pools. Note that due to accumulation of positive mutations, the latter may increase over iterations of the cycle, effectively lowering the transmission barrier between the pools (Fig. 1b).

Over time and in the right environment this process can lead to accumulation of multiple positive mutations, not unlike those reported for the Omicron variant. The characteristic parameters of the pools interacting in the cycle, such as size, rates, density and others can be determined when specific properties of the variant are known with better confidence.

\section{Discussion}

Interactions of immunity induced by mass vaccination with rapidly evolving viral agents can produce complex epidemiological scenarios, including, in some specific situations and environments, accelerated production of resistant strains. One such scenario, based on positive reinforcement interactions of two pools of population, is described in this work as a possible mechanism of accumulation of positive mutations leading to emergence of variants similar to Omicron.

An indirect support for a mechanism of this type is provided by another variant with a large number of mutations reported recently [9]. Further studies in the communities where these variants originated may provide essential inputs for validation or rejection of the hypothesis of accelerated variant production discussed in this work.

It remains to be confirmed by further research whether Omicron emerged through such a mechanism, a similar one or in an entirely different way, certainly an interesting and challenging direction for further investigation.

In the meantime, strategies based on selective vaccination, effective early screening for possible complications, effective treatments that are entering clinical practice $[10,11]$ and environmental methods of suppression of the infectious spread $[12,13]$ may offer a direction toward sustainable coexistence with Covid and eventual return to normal condition in the societies.

\section{References}

1. World Health Organization: Classification of Omicron (B.1.1.529): SARS-CoV-2 Variant of Concern. WHO, https://www.who.int/news/item/26-11-2021-classification-of-omicron(b.1.1.529)-sars-cov-2-variant-of-concern (29.11.2021).

2. Parekh M., Platt P., Team, Global Health Security, Barnes J.: Coronavirus latest news: EU suspends all flights to southern Africa over omicron Covid variant fears. The Telegraph, 26.11.2021.

3. Callaway E.: Heavily mutated Omicron variant puts scientists on alert. Nature News, 27.11.2021.

4. Japan confirms first case of omicron coronavirus variant. The Japan Times, 30.11.2021.

5. Omicron variant now detected in North America Canadian officials say. NBC News, 28.11.2021.

6. Dolgikh S.: Immuno-alignment effect due to mass DIV immunization in rapidly evolving viral environments. Cambridge Open Engage. doi: 10.33774/coe-2021-8b07r (2021). 
7. Covid: Dozens test positive on SA-Netherlands flights. BBC News, 27.11.2021.

8. Fully vaccinated NHL team in Ottawa forced to cancel games due to COVID outbreak. National Post, 18.11.2021.

9. Colson P., Delerce J., Burel E., Dahan J., Jouffret A. et al.: Emergence in Southern France of a new SARS-CoV-2 variant of probably Cameroonian origin harbouring both substitutions N501Y and E484K in the spike protein. medRxiv doi: 10.1101/2021.12.24.21268174 (2021).

10. U.S. Food \& Drug Administration: Coronavirus (COVID-19) update: FDA authorizes First oral antiviral for treatment of COVID-19. 22.12.2021.

11. Merck: Merck and Ridgeback's molnupiravir receives U.S. FDA emergency use authorization for the treatment of high-risk adults with mild to moderate COVID-19. 23.12.2021.

12. Tabish M., Khatoon A., Alkahtani S. et al.: Approaches for prevention and environmental management of novel COVID-19. Environmental Science and Pollution Research, 28, 40311-40321 (2020).

13. Dolgikh, S.: Smart-Covid: intelligent solutions for higher risk environments, HAL archives-ouvertes, hal-02915459 (2020). 